\title{
Tuberculosis, one disease, many faces
}

\author{
G.B. Migliori1, R. Centis ${ }^{1}$, C. Lange², A. Matteelli3, D. Cirillo4
}

Monaldi Arch Chest Dis 2008; 69: 1, 2-4.

1 WHO Collaborating Centre for TB and Lung Diseases, Fondazione S. Maugeri, Care and Research Institute, Tradate, Italy.

2 Division of Clinical Infectious Diseases, Medical Clinic, Research Center Borstel, Borstel, Germany.

3 University of Brescia, Brescia, Italy.

4 Emerging Bacterial Pathogens Unit, S. Raffaele Scientific Institute, Milano, Italy.

Correspondence: G.B. Migliori, WHO Collaborating Centre for TB and Lung Diseases, Fondazione S. Maugeri, Care and Research Institute/ TBNET Secretariat (TuBerculosis Network in Europe Trialsgroup)/ Stop TB Italy, via Roncaccio 16, 21049, Tradate (Va), Italy; e-mail: giovannibattista.migliori@fsm.it

Tuberculosis (TB) is a disease with multiple faces. It can be prevented (by way of vaccination), treated and cured. The best intervention to fight TB today is treatment of sputum smear positive, infectious cases[1]. Its transmission and trends follow well defined modelling patterns [1-4], its immunology represents a unique study model [5]. TB affected pre-historical mammouths and Egyptian mummies, survived industrialisation and globalisation. Although the introduction of modern antiTB chemotherapy and implementation of effective TB control programmes [6-7] contributed to reduce mortality and morbidity at country level, global TB incidence continued to grow till 2007 taking advantage of the HIV pandemic as well as all the conditions and social factors promoting reduced immunity, e.g. poverty, wars, malnutrition and other co-morbidities) [8-10].

With nearly 9 million new cases and over 2 million deaths per year in 2007, TB is a public health priority on a global level. The $4^{\text {th }}$ Drug Resistance Survey report published a few days ago [11] indicates that MDR - and XDR (multidrug-resistant and extensively drug-resistant)-TB represent a global emergency.

The highest rate of MDR-TB was detected in Baku, the capital of Azerbaijan, where 22.3 percent of new tuberculosis cases were resistant to the standard anti-tuberculosis drug regimen during the survey period from 2002 to 2006. Alarmingly high rates had also been detected in Moldova (19.4 percent), Donetsk in the Ukraine (16 percent), Tomsk Oblast in Russia (15 percent) and Tashkent in Uzbekistan (14.8 percent). 45 Countries had already notified at least one case of XDR-TB.

Monaldi Arch Chest Dis paid specific attention to this disease, through the dedicated section "TB Corner" as well as a qualified choice of peer-reviewed scientific articles.

The aim of this Editorial is to introduce and comment on three TB papers [12-14] focusing on new and stimulating aspects of TB research and control. These papers give us the opportunity to discuss the "hot" areas presently attracting the readers' interest, namely the role and contribution of IGRA (interferon-gamma release assay) tests, genomics and proteinomics, and the problems of controlling the disease in the context of high HIV and MDR/XDR prevalence situations.

The manuscript by I. Gerogianni [12] raises the interesting hypothesis that IFN.gamma predicts the development of residual pleural thickening in TB pleurisy, as observed in 25 out of the 31 patients studied. Furthermore the manuscript describes the epidemiology of pleural thickening and the criteria to define it, as well as the immunological weapons to diagnose it.

The review by D. Lykouras [13] discusses the role of human genes in modulating the immune response against TB infection.

Finally, the TB Corner section presents the unusual association between Mycobacterium tuberculosis and Aspergillus fumigatus [14].

There are five main updates relevant to global TB control [7]:

First, in 2006, the new Stop TB Strategy was launched by the World Health Organisation (WHO), following intensive exploration and discussion with TB control programme managers in high burden countries, and partner organisations, including technical agencies and donors [7]. The DOTS strategy (consisting of five key elements: government commitment, diagnosis through microscopy, standardised and supervised treatment, uninterrupted drug supply, and regular programme monitoring) that greatly contributed to improving global TB control over the last 10 years, was revisited to pursue DOTS expansion while making explicit five additional components that must be implemented to reach the 2015 Millennium Development Goals (MDGs) relevant to TB control. Thus, the Strategy consists of: 1) pursuing highquality DOTS expansion and enhancement; 2) addressing TB/HIV, multidrug-resistant TB and other challenges; 3 ) contributing to strengthening the health system; 4) engaging all care providers; 5) 
empower people with TB and communities; and 6) enable and promote research. The MDG 6 relevant to TB aims at combating HIV, malaria and other diseases. Its target 8 consists of halting by 2015 and beginning to reverse incidence of disease; its indicator 23 consists of TB prevalence and mortality; and its indicator 24 is the proportion of cases detected and cured under DOTS.

Secondly, the new Global Plan to Stop TB, 2006-2015 was launched in January 2006. The plan, underpinned by the Stop TB Strategy, describes strategies, financial requirements and existing gaps to reach the MDGs in all regions of the world. It identifies the need for 56 billion US\$ over the decade, of which 47 billion is for implementation of country programmes, including activities related to HIV-associated $\mathrm{TB}$, the approach to MDR-TB (formerly referred to as "DOTS-Plus"), and the engagement of non-state providers; and the remaining 9 billion for research and development of new tools. Considering the significant prevalence of MDR and XDR cases in several countries of the former Soviet Union, Europe will benefit from $20 \%$ of this budget although it contributes to only $5 \%$ of the global burden of TB cases.

Thirdly, new International Standards of TB Care (ISTC) have been published and widely distributed, to guide both public and private health care providers to deliver quality DOTS services.

Fourthly, the new WHO guidelines on drug-resistant TB management have been published, stressing the importance of a proper use of secondline drugs required to treat drug-resistant and MDR-TB cases.

Finally, 2006 will also be remembered for the appearance of XDR-TB, a very serious form of TB against which our armamentarium has virtually lost all its most powerful ammunition. XDR$\mathrm{TB}$ is defined as resistant to at least rifampicin and isoniazid (multidrug-resistant or MDR-TB) plus to any fluoroquinolone and at least one of three injectable anti-TB drugs (capreomycin, kanamycin, or amikacin) [6]. Preliminary reports from a number of sources following the XDR-TB outbreak in Tugela Ferry, Kwa Zulu Natal, South Africa suggested that XDR-TB may be almost incurable [15-17]. The XDR-TB-defining drugs are those considered essential to achieve successful outcomes in MDR-TB cases. As XDR-TB is still a newly described disease, evidence available on the role of the different classes of anti-TB drugs in its treatment is scant and slow to accumulate. Italian research, through TBNET (TuBerculosis Network in Europe Trialsgroup) demonstrated that XDR-TB patients have a higher risk of death and failure than those with MDR-TB [15], and i) resistance to additional first-line drugs (other than isoniazid and rifampicin) is a predictor of adverse outcomes [18]; ii) resistance to fluoroquinolones contributes to increased risk of death and failure in these cases [19]; iii) resistance to second line injectable drugs (and capreomycin in particular) is also associated with increased unfavourable outcomes [20].
In the past decade, major advances have been achieved in the diagnosis of tuberculosis, yet advances in prevention by a better vaccine or in treatment by the development of novel drugs are not foreseeable in the near future.

A collaborative effort is necessary to coordinate the best energies available to improve TB prevention and treatment in order to win the battle against the white plague.

\section{References}

1. Rieder HL. Epidemiologic basis of tuberculosis control. International Union Against Tuberculosis and Lung Disease, Paris: 1999.

2. Dye C. Breaking a law: tuberculosis disobeys Styblo's rule. Bull World Health Organ 2008; 86: 4

3. Van Leth F, Van der Werf MJ, Borgdorff MW. Prevalence of tuberculous infection and incidence of tuberculosis: a re-assessment of the Styblo rule. Bull World Health Organ 2008; 86: 20-6.

4. Dye C, Bassili A, Bierrenbach A, Broekmans J, et al. Measuring tuberculosis burden, trends, and the impact of control programmes. Lancet Infect Dis 2008 Jan 15; [Epub ahead of print]

5. Rook GA, Zumla A. Advances in the immunopathogenesis of pulmonary tuberculosis. Curr Opin Pulm Med 2001; 7: 116-123.

6. Cox HS, Morrow M, Deutschmann PW. Long term efficacy of DOTS regimens for tuberculosis: systematic review. BMJ 2008 Feb 4; [Epub ahead of print].

7. Migliori GB, Loddenkemper R, Blasi F, Raviglione MC. 125 years after Robert Koch's discovery of the tubercle bacillus: the new XDR-TB threat. Is "science" enough to tackle the epidemic? Eur Respir J 2007; 29: 423-427.

8. Cegielski PJ, Kohlmeier L, Cornoni-Huntley J. Relative and Attributable Risks of Tuberculosis due to Undernutrition in a Population-based Sample of Adults. TSRU progress report 2007. The Hague: KNCV, 2007.

9. Lienhardt C. From exposure to disease: the role of environmental factors in susceptibility to and development of tuberculosis. Epidemiologic Reviews 2001; 23: 288-301.

10. WHO. Commision on Social Determinants of Health. WHO/EIP/EQH/01/2006. Geneva: World Health Organization, 2006.

11. World Health Organization (WHO). Anti-tuberculosis drug resistance in the world: the WHO/IUATLD Global Project on Anti-Tuberculosis Drug Resistance Surveillance. Geneva, Switzerland: WHO/HTM/TB/2008.394

12. Gerogianni I, Papala M, Tsopa P, et al. Could IFN- $\gamma$ predict the development of residual pleural thickening in tuberculous pleurisy? Monaldi Arch Chest Dis 2008; 69: 18-23.

13. Lykouras D, Sampsonas F, Kaparianos A, Karkoulias K, Tsoukalas G, Spiropoulos K. Human genes in TB infection: their role in immune response. Monaldi Arch Chest Dis 2008; 69: 24-31.

14. Agarwal R, Singh N, Aggarwal A.N. An unusual association between Mycobacterium tuberculosis and Aspergillus fumigatus. Monaldi Arch Chest Dis 2008; 69: 32-34.

15. Migliori GB, Ortmann J, Girardi E, et al. Extensively drug-resistant tuberculosis, Italy and Germany. Emerg Infect Dis 2007; 13: 780-782.

16. Holtz TH, Cegielski JP. Origin of the term XDR-TB. Eur Respir J 2007; 30: 396.

17. Gandhi NR, Moll A, Sturm AW et al. Extensively drug- 
resistant tuberculosis as a cause of death in patients coinfected with tuberculosis and HIV in a rural area of South Africa. Lancet 2006; 368: 1575-1580.

18. Migliori GB, Besozzi G, Girardi E, et al. Clinical and operational value of the extensively drug- resistant tuberculosis definition. Eur Respir J 2007; 30: 623-626.
19. Migliori GB, Lange C, Girardi E, et al. Fluoroquinolones: are they essential to treat multidrug-resistant tuberculosis? Eur Respir J 2008; 31: in press.

20. Migliori GB, Muetterlein R, Sotgiu G, et al. Secondline injectables: are they essential to treat MDR/XDRTB cases? Eur Respir J 2008, abstract submitted.

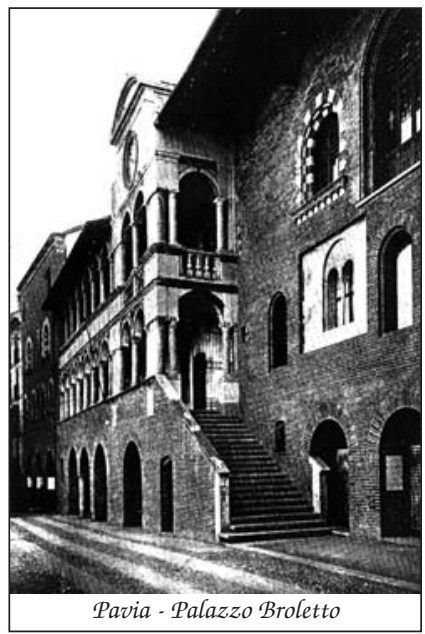

